\title{
A Grid-Enabled Scene Rendering Application
}

\author{
M. Caballer, V. Hernández, and J.E. Román \\ D. de Sistemas Informáticos y Computación, Universidad Politécnica de Valencia, \\ Camino de Vera, s/n, E-46022 Valencia, Spain. \\ \{micafer, vhernand, jroman\}@dsic.upv.es
}

\begin{abstract}
The work presented in this paper represents a step forward in the wide adoption of grid technologies in more conventional commercial applications. The application treated herein is a global illumination scene rendering engine in which grid tools have been used to execute the computation remotely, as well as to incorporate certain interesting characteristics. This engine can be incorporated, for instance, in an electronic commerce application in order to add visualization capabilities to a conventional virtual store. The rendering engine implements a parallel version of the radiosity illumination algorithm, providing high quality and allowing a fast response for moderately complex scenes.
\end{abstract}

\section{Introduction}

This paper presents a remote scene rendering service, implemented with standard grid tools. These tools provide some desirable features such as uniformity, transparency, reliability, ubiquity and security [4] which can be very important in some applications but are lacking in more conventional Internet technologies. In particular, Globus Toolkit 2 3 has been selected for the middleware layer, since it is currently one of the most widely accepted grid frameworks.

This work is a follow-up of the EU-funded research project VRE-Commerce (see http://www.grycap.upv.es/vrecommerce), whose main objective was the integration of fast synthetic image generation in electronic commerce applications, with special focus on the furniture and ceramics sectors. The application enables the user to obtain realistic renders of customized environments in which products from the virtual store have been placed. These images are generated with radiosity techniques [6], in particular with a parallel implementation so that the response time is bearable for an e-commerce setting (see [2] for details).

\section{Prototype Implementation}

The complete operation of the application is illustrated in Figure 1 The user accesses the virtual store located in the web server and makes the image generation request (step 1). A CGI program (linked with the Globus libraries) located in the web server is in charge of managing this request on behalf of the user and having it processed by the grid. The required steps are described next. In this 


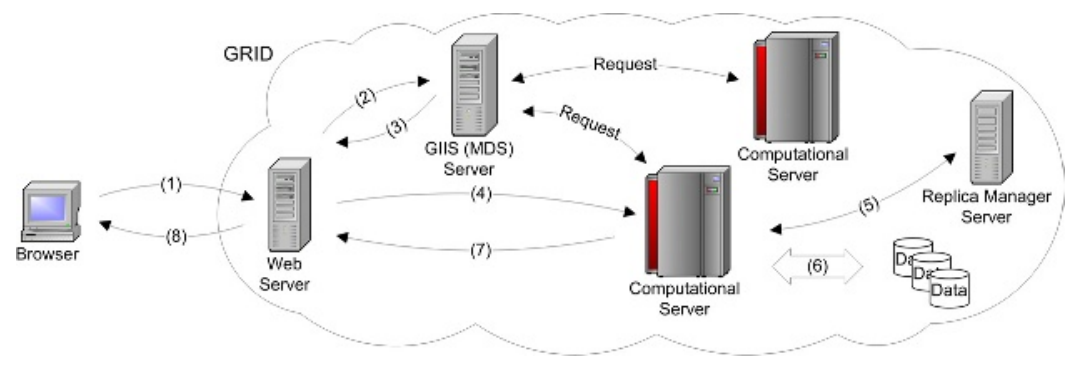

Fig. 1. Scheme of the rendering service.

process, several different Globus components participate, which may be placed in different physical locations.

Discovery of Grid Resources. First, the Monitoring and Discovery Service (MDS) included in Globus is used to obtain information about the resources available in the grid. The CGI program uses the information provided by MDS in order to select the most suitable resource. The decision is based on the number of free nodes. This information is also used to select the number of nodes to allocate for the radiosity program. If there are not enough free resources, the program will suggest the user to wait and try later (steps 2 and 3).

Job Submission. Once the computational server has been chosen, the CGI program launches the radiosity calculation via the Globus Resource Allocation Manager (GRAM). Before this, it is necessary to authenticate within the grid by means of a proxy, containing the certificate and public key of the grid user. Once authenticated, the job is submitted to the remote resource (step 4).

Update of Geometrical Data Files. The radiosity program needs a set of files containing the geometrical models of furniture pieces or the tiles textures in the case of ceramics. These files belong to the different manufacturers that offer products in the application. The files are cached locally in each computational server. The grid scheme adds the possibility to update the master copies of the files by the manufacturers, as well as to add new ones. The program analyzes the scene looking for the furniture or ceramic pieces used, and then queries the Replica Manager for their location (step 5). Then the dates of remote and local files are compared so that download is only done if necessary (step 6).

Return of Results. The CGI program uses the identifier returned by the GRAM service to detect the job finalization. When the job is finished the program uses the I/O functions provided by the GASS (Global Access to Secondary Storage) service to send the image generated by the radiosity algorithm back to the web server (step 7). Using these functions the CGI program can obtain the image with no need of modification of the radiosity code. Finally, the web server will display the image in the client's web browser (step 8). 

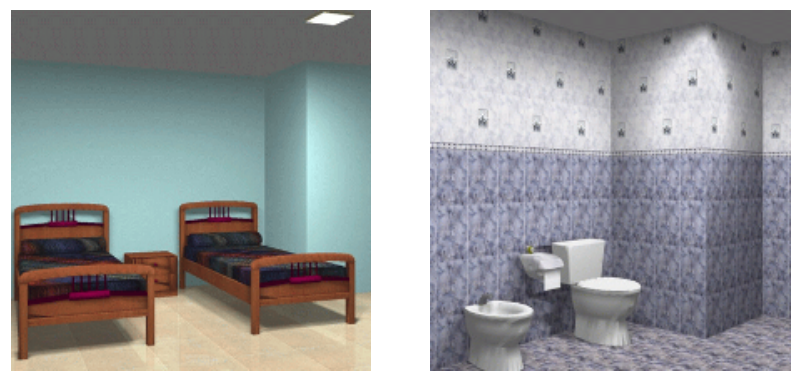

Fig. 2. Some of the images used for validation and timing.

\section{Testing and Results}

Several tests have been carried out to verify the operation and performance of the whole system. Four test cases have been used to measure times in order to assess the overhead incurred by the Globus components. The chosen scenes are representative examples of the scenes that can be designed with the application. The generated images are illustrated in Figure 2

The measured time begins when the user clicks the "Generate Image" button and finishes when the system starts to send the resulting image (the transfer time of the image is not taken into account because it depends on the type of Internet connection). The times considered for comparison have been the time consumed by the radiosity program and the accumulated time corresponding to the grid functions (which includes steps $1-5$ and 7 in figure 1). The time obtained for each of the test cases is shown in Table 1 along with the initial number of polygons and patches at the end of execution, as well as the number of lights (to give an indication of the size of the problem).

The time added by the grid operations is slightly larger than 10 seconds, which is reasonable considering the response time requirement of $1-2$ minutes established initially in the VRE-Commerce project. Although the time consumed by Globus functions represents a non-negligible percentage, it is constant, and, therefore, it will be less significant in more complex scenes. More details about the tests can be found in [1].

Table 1. Characteristics of the test scenes and measured times (in seconds).

\begin{tabular}{|l|c|c|c|c|c|c|}
\hline Scene & Polygons & Patches & Lights & Radiosity & Globus & Total \\
\hline Ex. 1 & 2426 & 28562 & 2 & 46 & 12 & 59 \\
\hline Ex. 2 & 5160 & 28740 & 2 & 56 & 12 & 68 \\
\hline Ex. 3 & 4908 & 24372 & 2 & 60 & 11 & 71 \\
\hline Ex. 4 & 1374 & 20118 & 2 & 17 & 13 & 30 \\
\hline
\end{tabular}




\section{Conclusions and Future Work}

Grid tools pursue a number of properties (ubiquity, security, etc.) that are very important for distributed applications. Globus Toolkit 2 provides all the functionality required for our application. The benefits obtained by applying grid technologies have been the following in this case:

- Scalability: it is possible to add computational resources easily, so that the application can automatically use them.

- Fault tolerance: if any computational server fails, the system can redirect the request to the active ones.

- Security: the user must be authenticated to access the system, and all network data is encrypted. Although in this application there are no confidential data, security may be a key issue in other commercial applications.

- Load balancing: the system selects the most suitable grid resource.

- Data management: the system enables easy update of the furniture data and ceramic textures.

The system operation has been tested successfully in different situations, showing a robust behavior against possible failures. The execution overheads, owing to Globus components, are reasonable for the application.

With respect to future development of the system, different working lines are being considered. On one hand, after the release of Globus Toolkit 3, the logical extension would be to migrate to this new platform to take advantage of its new possibilities, adapting the application to the Open Grid Services Architecture defined in [5]. Among other things, this involves standardizing the way information is exchanged between the client application and the grid service. This opens the door to providing service to other applications such as standalone room design tools or, more generally, anyone requiring high-quality, fast rendering. For certain applications such as film making, this would entail harnessing greater amount of computing power and using geographically distributed resources. Finally, a desirable feature is to represent resulting renders with a 3-D modeling language (such as VRML or X3D) to allow for interactivity.

\section{References}

1. Caballer M., Hernández V., Román J.E.: A grid-enabled scene rendering application. Technical Report DSIC-II/04/04, Universidad Politécnica de Valencia (2004)

2. Caballer M., Guerrero D., Hernández V., Román J.E.: A parallel rendering algorithm based on hierarchical radiosity. Lecture Notes in Computer Science 2565 (2003) 523-536

3. Foster I., Kesselman C.: Globus: A metacomputing infrastructure toolkit. Internat. J. Supercomputer Appl. 11 (1997) 115-128

4. Foster I., Kesselman C.: The Grid: Blueprint for a New Computing Infrastructure. Morgan Kaufmann (1998)

5. Foster I., Kesselman C., Nick J., Tuecke S.: The physiology of the grid: An open grid services architecture for distributed systems integration (2002)

6. Sillion F., Puech C.: Radiosity and Global Illumination. Morgan Kaufmann (1994) 